\title{
PLUCKED-STRING SYNTHESIS ALGORITHMS WITH TENSION MODULATION NONLINEARITY
}

\author{
Vesa Välimäki, Tero Tolonen, and Matti Karjalainen \\ Helsinki University of Technology, Laboratory of Acoustics and Audio Signal Processing \\ P.O. Box 3000, FIN-02015 HUT, Espoo, Finland \\ E-mail: Vesa.Valimaki@hut.fi,Tero.Tolonen@hut.fi, Matti.Karjalainen@hut.fi \\ http://www.acoustics.hut.fi/
}

\begin{abstract}
Digital waveguide modeling of a nonlinear vibrating string is investigated when the nonlinearity is essentially caused by tension modulation. We derive synthesis models where the nonlinearity is implemented with a time-varying fractional delay filter. Also, conversion from a dual-delay-line physical model into a single-delay-loop model is explained. Realistic synthetic tones with nonlinear effects are obtained by introducing minor amendments to a linear string synthesis algorithm. It is shown how synthetic plucked-string tones are modified as a consequence of tension modulation. Examples of synthesized tones are available at http://www.acoustics.hut.fi/ ttolonen/sounddemos/tmstr/.
\end{abstract}

\section{INTRODUCTION}

In physical modeling of plucked and struck string instruments, the vibration of a string is typically simulated with a linear system, such as a digital waveguide model [9], [3]. However, a vibrating string is linear only to a first approximation, and consequently, nonlinear phenomena exhibited by every real string, such as the modulation of string tension, are inherently omitted in these simulations. Perceptually, the two most important phenomena caused by tension modulation are the pitch variation depending on the vibration amplitude and the generation of missing harmonics due to nonlinear coupling of the vibration modes. While the nonlinearity has been studied both analytically and experimentally [1], [6], [5], to our knowledge the only synthesis model of a vibrating string incorporating tension modulation has been proposed by Karjalainen et al. [2], who used a memoryless nonlinearity. Synthesis models have been presented previously for a nonlinearly terminated string [8] and for the trombone where nonlinear wave propagation takes place [7]. Recently, we showed that these two cases can be generalized to signal-dependent nonlinearities that can be implemented using a time-varying fractional delay (TVFD) filter with a signaldependent delay parameter [11].

In this paper, we first discuss the nonlinear string in Section 2 where we also show analysis results of recorded guitar tones. In Section 3, we propose a new digital waveguide synthesis model for the nonlinear string, which utilizes the TVFD structure. We also present a computationally efficient implementation structure. In particular, we show that with little compromises in the simulation accuracy, we may obtain a single-delay-loop model that is much cheaper in terms of computational complexity than the dual-delay-line model. In Section 4, we illustrate by synthesis examples that our nonlinear string simulation produces synthetic tones which are more realistic than those produced with linear models.

\section{NONLINEAR STRING VIBRATIONS}

\subsection{Tension Modulation}

The primary cause of the nonlinearity of a vibrating string is the modulation on the string length; any transversal displacement of the string results in an increase to its length. We consider one polarization of the transversal string vibration only. We also assume that the propagation speed of longitudinal vibration is much larger than that of transversal vibration so that the increase in tension caused by prolongation is immediately spread over the whole string. The tension along the string, $F_{\mathrm{t}}$, can be expressed as [5]

$$
F_{\mathrm{t}}=F_{\text {nom }}+\frac{E S \ell_{\mathrm{dev}}}{\ell_{\text {nom }}}
$$

where $F_{\text {nom }}$ is the nominal tension of the string at rest, $E$ is Young's modulus, $S$ is the cross-sectional area of the string, $\ell_{\text {nom }}$ is the nominal string length, and $\ell_{\text {dev }}$ is its deviation, i.e.,

$$
\ell_{\mathrm{dev}}=\int_{0}^{\ell_{\mathrm{nom}}} \sqrt{1+\left(\frac{\partial y}{\partial x}\right)^{2}} d x-\ell_{\mathrm{nom}}
$$

where $y$ is the displacement of the string and $x$ is the spatial coordinate along the string (see, e.g., [5]).

The tension modulation is illustrated in Fig. 1 for two cases where a string is ideally plucked in the middle (top) and at a distance $0.19 \ell$ nom from the termination (bottom). In this simulation the string is assumed to have rigid end supports. The nominal string tension $F_{\text {nom }}$ is depicted with a dashed line. The monotonic decaying curve is the average tension that has been computed with a running time average over each period of the tension curve. Note that in the upper figure the tension equals $F_{\text {nom }}$ once during each period whereas in the lower figure the tension only approaches $F_{\text {nom }}$ with time.

The tension modulation affects the transversal speed $c$ as

$$
c=\sqrt{\frac{F_{\mathrm{t}}}{\rho}}
$$

where $\rho$ is the linear mass density of the string. The fundamental frequency of the tone is linearly related to the average wave propagation speed. Since the average wave speed depends on the average tension (examples shown in Fig. 1), the fundamental frequency is expected to decay exponentially towards the nominal value. Note that it is impossible to directly observe the oscillating tension in a tone recorded from a real string, rather, only the decaying fundamental frequency may be perceived or revealed through signal analysis.

The wave propagation speed of Eq. (3) can be rewritten as 


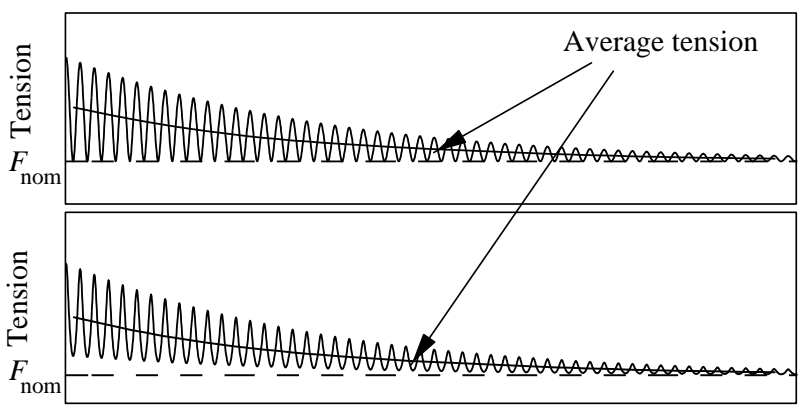

Time

Figure 1. Tension variation of a simulated vibrating string (solid line) for two different plucking points. The tension of the string at rest, $F_{\text {nom }}$, is indicated with a dashed line.

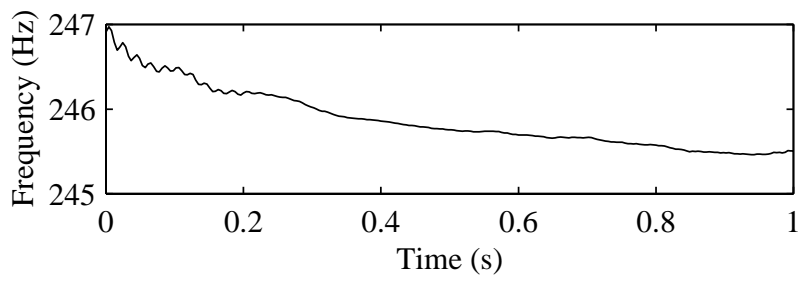

Figure 2. Time history of the fundamental-frequency estimate of an electric guitar tone.

$$
\begin{aligned}
c & =\sqrt{\frac{F_{\mathrm{t}}}{\rho}}=\sqrt{\left(\frac{\ell+\ell_{\mathrm{dev}}}{\rho_{\mathrm{nom}} \ell}\right)\left(F_{\mathrm{nom}}+\frac{E S \ell_{\mathrm{dev}}}{\ell}\right)} \\
& =c_{\text {nom }} \sqrt{1+\left(1+\frac{E S}{F_{\mathrm{nom}}}\right) \frac{\ell_{\mathrm{dev}}}{\ell}+\frac{E S}{F_{\text {nom }}}\left(\frac{\ell_{\mathrm{dev}}}{\ell}\right)^{2}}
\end{aligned}
$$

where the mass density $\rho$ and the nominal wave speed $c_{\text {nom }}$ are defined as follows:

$$
\rho=\rho_{\text {nom }} \ell /\left(\ell+\ell_{\mathrm{dev}}\right) \text { and } c_{\text {nom }}=\sqrt{F_{\text {nom }} / \rho_{\text {nom }}}
$$

where $\rho_{\text {nom }}$ is the nominal mass density when the string is at rest.

In addition to the decay of fundamental frequency, the nonlinear vibration provides a mechanism for coupling of the vibration modes assuming that at least one of the end supports is not completely rigid [5]. This allows generation of harmonics that are initially missing due to initial conditions [5]. In practice, the string terminations allow this kind of nonlinear mode coupling in every musical instrument. In the case of the kantele, a traditional Finnish plucked string instrument, the nonlinearity caused by a yielding tuning peg has been analyzed and modeled by Karjalainen et al. [2] who used a memoryless nonlinear model.

\subsection{Analysis of Recorded Guitar Tones}

Figure 2 shows the fundamental-frequency trajectory of a tone played with an electric guitar with steel strings. This curve was produced using a pitch-synchronous short-time autocorrelation analysis. The recorded signal was divided into analysis frames that are four times longer than the nominal fundamental period. The frames were windowed using a Hamming window of the same length. The consecutive analysis frames overlap by $50 \%$. A high-resolution fundamental-frequency estimate of each frame was computed by first searching for the maximum of the autocor-

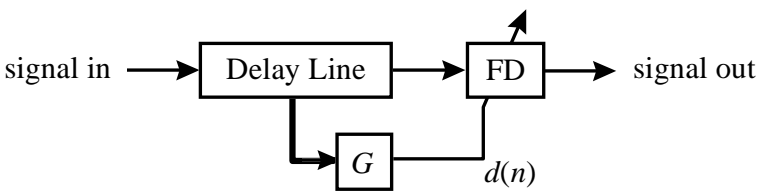

Figure 3. General nonlinear delay line where the delayline signal controls the delay parameter $d(n)$ of a fractional delay (FD) filter (adopted from [11]). Function $G$ computes $d(n)$ based on the contents of the delay line.

relation function and by fine-tuning this initial estimate using parabolic interpolation. Figure 2 exhibits a clear exponential decay of the fundamental frequency. The decay within a time interval of one second is approximately $1.5 \mathrm{~Hz}$. This time series may be used to obtain parameters for a discrete-time simulation of the nonlinear string.

\section{TENSION MODULATION MODELS}

Our approach to discrete-time simulation of tension modulation is to extend the bidirectional linear waveguide model [9], [3] with a signal-dependent nonlinear TVFD structure discussed in [11]. The two delay lines are implemented using two nonlinear delay lines of Fig. 3. The function $G$ maps the signal in the delay line into a delay parameter $d(n)$ where $n$ is the discrete time index. The delay parameter is used to control the FD filter. Each delay line models the wave propagation delay $\tau / 2$ between the two ends of the string, which is determined as

$$
\tau=\lambda / c
$$

where $\lambda$ is twice the distance between the ends of the string, and $c$ is the wave propagation speed as given by Eq. (3). The delayline length in sampling intervals is obtained as

$$
L=f_{\mathrm{s}} \tau
$$

The tension modulation can be readily formulated according to the general framework of Fig. 3, since the tension variation is a function of only the deviation of the string length. In the following, we first derive a dual-delay-line string model with tension modulation using the TVFD structure and then, with minor compromises, simplify it into a computationally more efficient single-delay-loop string model. We utilize the formulation of a TVFD structure for uniformly distributed time-varying propagation velocity described in [11]. This is applicable to simulation of tension modulation, since the propagation speed of transversal vibrations is approximately uniform due to the high propagation speed of longitudinal vibrations.

\subsection{Dual-Delay-Line String Model}

The deviation of the delay-line length in samples can be approximated from the digital waveguide model as follows (cf. Eq. 2)

$$
\frac{L_{\mathrm{dev}}(n)}{2}=\sum_{k=0}^{L_{\mathrm{nom}} / 2-1} \sqrt{1+\left[s^{+}(n, k)+s^{-}(n, k)\right]^{2}}-\frac{L_{\mathrm{nom}}}{2}
$$

where $s^{+}(n, k)$ and $s^{-}(n, k)$ are the slope signals traveling in the delay lines, $L_{\text {nom }}$ is the nominal delay-line length, and $n$ and $k$ are the discrete time and space indices, respectively. While this formula could be readily implemented, it can still be simplified. When we assume that $\left[s^{+}(n, k)+s^{-}(n, k)\right]^{2}<<1$, we may develop a Taylor approximation of the square root function. When the second and higher-order terms are excluded, this yields 


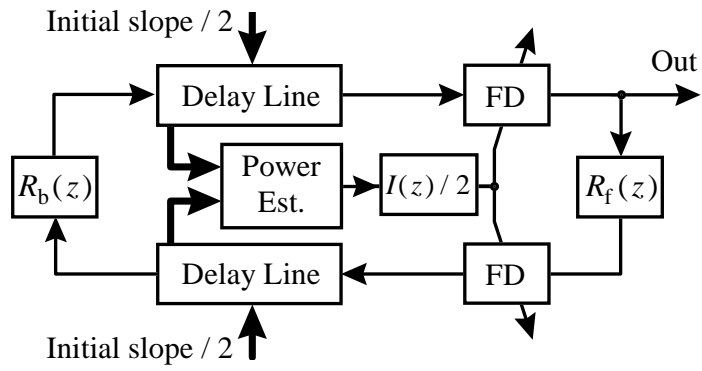

Figure 4. Dual-delay-line string synthesis model with tension modulation implemented with signal-dependent fractional delay elements.

$$
L_{\mathrm{dev}}(n) \approx \sum_{k=0}^{L_{\mathrm{nom}} / 2-1}\left[s^{+}(n, k)+s^{-}(n, k)\right]^{2}
$$

where we have multiplied both sides by 2 and the term $L_{\text {nom }}$ is canceled by the sum of the constant terms of the Taylor series.

Using Eqs. (5) and (6), we may write

$$
L(n)=f_{\mathrm{s}} \lambda / c(n)
$$

where $c(n)$ can be extended according to Eq. (4), which we may approximate by a Taylor series. Neglecting second and higherorder terms and substituting into Eq. (9), we obtain

$$
L(n) \approx \frac{f_{\mathrm{s}} \lambda}{c_{\mathrm{nom}}}\left\{1-\frac{1}{2}\left[(1+K) \frac{L_{\mathrm{dev}}(n)}{L_{\mathrm{nom}}}+K\left(\frac{L_{\mathrm{dev}}(n)}{L_{\mathrm{nom}}}\right)^{2}\right]\right\}(10)
$$

where $K=E S / F_{\text {nom }}$ and we have replaced the ratio $\ell_{\text {dev }} / \ell$ with $L_{\mathrm{dev}}(n) / L$.

Looking at Eqs. (10) and (8), we now see that the string tension modulation can be simulated by controlling a fractional delay filter with a power-like signal $L_{\mathrm{dev}}(n)$ which is a sum of the pair-wise squared sums of the delay-line signals (see Eq. (8)).

The model structure illustrated in Figure 4 implements a version of this algorithm where $L_{\mathrm{dev}}(n)$ is computed using a powerestimation algorithm, and we have dropped the second-order term in Eq. (10) and aggregated all the terms multiplying $L_{\mathrm{dev}}(n)$. In addition, we have inserted a one-pole filter that approximates temporal integration. It implements memory that is required when the distributed time-varying resampling is lumped into a single point at the end of the delay line (see [11], Section 3.2 for details). The transfer function of the one-pole filter is

$$
I(z)=-g_{\mathrm{p}} \frac{1+a_{\mathrm{p}}}{1+a_{\mathrm{p}} z^{-1}}
$$

where coefficient $a_{\mathrm{p}}$ controlling the bandwidth of integration is chosen to be $-1<a_{\mathrm{p}}<0$ and gain factor $g_{\mathrm{p}}$ that controls the depth of tension modulation must be nonnegative. When $g_{\mathrm{p}}=0$, the traditional linear digital waveguide models is obtained. When $g_{p}>0$, the nonlinear effect increases with the power of the delayline signal. Note that $I(z)$ also includes the minus sign so that when its input signal is a power-like, non-negative sequence, the output signal will be non-positive. As the output signal controls the length of the delay line, the delay is inversely proportional to the input signal of $I(z)$. Filters $R_{\mathrm{b}}(z)$ and $R_{\mathrm{f}}(z)$ in Figure 4 approximate the reflections from the terminations of the string. Note that a division by 2 is included in Figure 4 since the length

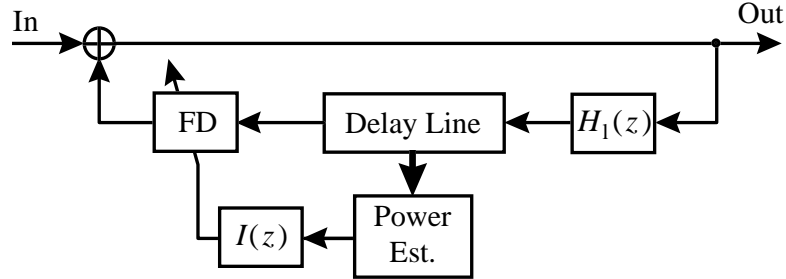

Figure 5. Single-delay-loop string synthesis model incorporating tension modulation.

variation is realized with two FD filters-one for each delay line.

In this application, a TVFD element is conveniently approximated using Lagrange interpolation [4], which is often used in digital waveguide models due to its easy coefficient update, bounded magnitude response that does not risk the stability of the feedback loop, and a transient-free behavior in time-varying cases. The Farrow structure can be used to implement a Lagrange interpolation so that its transfer function is directly controlled by a single parameter $d(n)$ [10], [11].

Above we have assumed that the nominal length of the string, $L_{\text {nom }}$, is an integral number. In general, the nominal length may be a non-integral multiple of the sampling interval. In such a case, the fractional part of the nominal delay-line length can be realized by using the same FD filter that is used for simulating tension modulation.

\subsection{Single-Delay-Loop String Model}

The structure developed above uses two delay lines (see Fig. 4). It would be desirable to use a single-delay-loop model instead due to computational savings. For a discussion on reasons for this, see, e.g., [3]. Here we derive an approximation of the above model that behaves similarly but is computationally cheaper.

First we may move one of the reflection filters over to the other side of the delay lines (to the left in Fig. 4) and consolidate it with the other reflection filter. This changes the contents of the delay lines little, since the magnitude of the reflection filters is very close to unity. The phase inversion that occurs with the reflection filter can be accounted for in the initial slope of the string by inverting one half of it. The two FD filters may also be combined into a single TVFD unit. This FD element is most conveniently placed at the end of the delay lines which may then be combined into a single delay line whose length will be equal to the total length of the two delay lines in the original model. The resulting single-delay-loop string model is presented in Fig. 5. Loop filter $H_{1}(z)$ now represents the composite lowpass filtering effect of $R_{\mathrm{b}}(z)$ and $R_{\mathrm{f}}(z)$.

The power estimation in Fig. 5 consists of summing the first sample of the delay line with last one, the second sample with the second last one, and so on, squaring all these sums and summing up all terms. The power estimation is the most time-consuming operation in the model. A simple way to reduce the computational burden of the squared sum is to approximate the instantaneous power as a sparse squared sum, where every $M$ th sample pair is included but the rest of the pairs are excluded. The sparse squared sum must be scaled by $M$ so that the magnitude of the instantaneous power is retained.

\section{SYNTHESIS EXAMPLES}

Examples of synthetic tones produced with the single-delay-loop model of Fig. 5 are presented in this section. In the examples, 

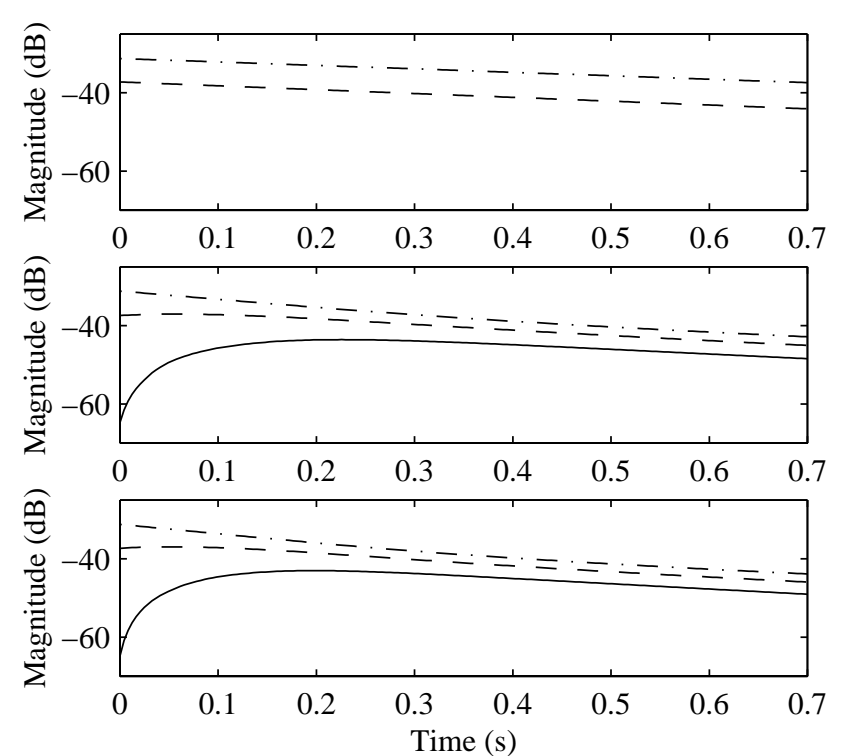

Figure 6. Envelopes of the first (dash-dot line), second (dashed line), and third (solid line) harmonic of synthetic tones generated by the linear (top), nonlinear singledelay-loop (middle) with $g_{\mathrm{p}}=10.0$, and the nonlinear single-delay-loop string algorithm with sparse power estimation $(M=6)$ and $g_{\mathrm{p}}=10.0$ (bottom).

$L_{\text {nom }}=90$ samples. The input signal of the model is the initial slope curve that causes a maximum displacement of 1 unit sample. The plucking point is $L_{\text {nom }} / 3$, and thus every third harmonic component is missing in tones synthesized with the linear model. Both the linear and the nonlinear cases are examined. A secondorder Lagrange interpolator is used as a TVFD filter.

Figure 6 shows the envelopes of three lowest harmonics of the synthetic tones as a function of time. In the linear case $\left(g_{\mathrm{p}}=0\right)$, the third harmonic is very weak (not visible in the top part of Fig. 6), as expected. In the case of the nonlinear model (Fig. 6, middle part) with $g_{\mathrm{p}}=10.0$, the third harmonic starts at a small level but soon its level begins to raise and finally it decays like the other harmonics. A synthetic tone produced with a sparse squared sum with $M=6$ has been analyzed on bottom in Fig. 6 . The harmonic envelopes have not changed much with respect to the middle part of Fig. 6 while the summing points have been reduced by $80 \%$ from 45 to 8 . This illustrates that the pruning of operations in power estimation does not necessarily deteriorate the output signal. Figure 7 presents the fundamental-frequency estimate of the two synthetic tones. Comparison against Fig. 2 suggests that the pitch variation is properly reproduced in both cases.

Synthetic string tones produced with the nonlinear model are perceived as less static than those generated with a linear model. When compared with original guitar tones, they sound more realistic. Examples of recorded and synthetic tones are available at http://www.acoustics.hut.fi/ ttolonen/sounddemos/tmstr/.

\section{SUMMARY}

This paper considered sound synthesis of plucked-string tones when the vibrating string behaves in a nonlinear fashion. The nonlinearity was assumed to be caused by tension modulation due to the non-negligible displacement of the string. This nonlinearity influences the frequencies and amplitudes of partials in

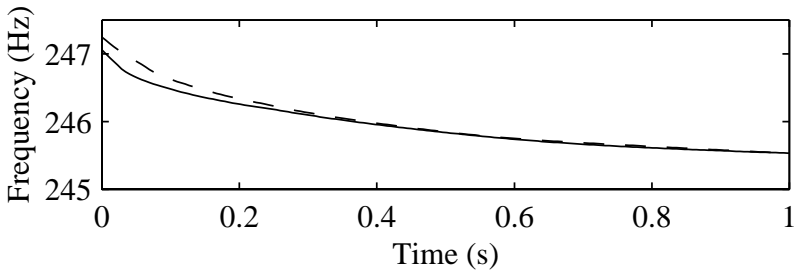

Figure 7. Fundamental frequency of the synthetic tone using standard (solid) and sparse squared sum (dashed).

tones produced by steel strings. A physics-based sound synthesis algorithm that is based around a digital waveguide model was derived which produces similar effects that are found in recordings of plucked-string tones. A computationally efficient simplified algorithm was also devised. Analysis of synthetic tones produced by the model revealed that the expected phenomena, that is, the nonlinear generation of missing harmonics and variation in the fundamental frequency, are reproduced.

\section{ACKNOWLEDGMENTS}

This work has been supported by the Academy of Finland and the Graduate School in Electronics, Telecommunications and Automation (GETA) at Helsinki University of Technology.

\section{REFERENCES}

[1] G. F. Carrier, "On the non-linear vibration problem of the elastic string,” Q. Appl. Math., vol. 3, pp. 157-165, 1945.

[2] M. Karjalainen, J. Backman, and J. Pölkki, "Analysis, modeling, and real-time sound synthesis of the kantele, a traditional Finnish string instrument," in Proc. IEEE ICASSP, vol. 1, pp. 229-232, Minneapolis, MN, 1993.

[3] M. Karjalainen, V. Välimäki, and T. Tolonen, "Pluckedstring models: from the Karplus-Strong algorithm to digital waveguides and beyond," Computer Music J., 22(3), 1998.

[4] T. I. Laakso, V. Välimäki, M. Karjalainen, and U. K. Laine, "Splitting the unit delay-tools for fractional delay filter design," IEEE Signal Processing Mag., 13(1): 30-60, 1996.

[5] K. A. Legge and N. H. Fletcher, "Nonlinear generation of missing modes on a vibrating string," J. Acoust. Soc. Am. 76(1): 5-12, 1984.

[6] P. M. Morse, Vibration and Sound. McGraw-Hill, 1948.

[7] R. Msallam, S. Dequidt, S. Tassart, and R. Caussé, "Physical model of the trombone including nonlinear propagation effects," in Proc. Int. Symp. Musical Acoust., vol. 2, pp. 419-424, Edinburgh, Scotland, Aug. 1997.

[8] J. R. Pierce and S. A. Van Duyne, "A passive nonlinear digital filter design which facilitates physics-based sound synthesis of highly nonlinear musical instruments," $J$. Acoust. Soc. Am., 101(2): 1120-1126, 1997.

[9] J. O. Smith, "Principles of digital waveguide models of musical instruments," in M. Kahrs and K. Brandenburg, Eds., Applications of Digital Signal Processing to Audio and Acoustics. Kluwer, 1998, chapter 10, pp. 417-466.

[10] V. Välimäki, "A new filter implementation strategy for Lagrange interpolation," in Proc. IEEE Int. Symp. Circuits and Systems, vol. 1, pp. 361-364, Seattle, WA, 1995.

[11] V. Välimäki, T. Tolonen, and M. Karjalainen, "Signaldependent nonlinearities for physical models using timevarying fractional delay filters," in Proc. Int. Computer Music Conf., Ann Arbor, MI, Oct. 1998. 